\title{
Au Nanoparticle Plasmon Sensor for Terpene Detection
}

\author{
Bin Chen, Chuanjun Liu, Manami Ota, and Kenshi Hayashi \\ Department of Electronics, Graduate School of Information Science and Electrical \\ Engineering, Kyushu University, 744, Motooka, Nishiku, Fukuoka 819-0395, Japan
}

\begin{abstract}
:
This paper describes a method to generate films composed of Au nanoparticles (AuNPs) assembled in a stepwise manner entirely from solution. AuNPs in the films were linked by two kinds of dithiol: 1,4benzenedithiol and 1,10-decanedithiol which have different molecular length and molecular electrical conductivity. Through comparing their LSPR spectra characters, we obtain that the film structures are different, and the difference is caused by structural characters of linkers. Layer-by-layer structured AuNPs films were used to detect terpene gases possessing different characters, and the local refractive index sensitivity improvement was realized. Sensitivity is attributed to the solubility between terpene gases and dithiol linkers.
\end{abstract}

Key words: AuNPs, LSPR, terpene detection,dithiol.

\section{Introduction}

Plants synthesize and emit a large variety of volatile organic compounds (VOCs) during their normal growth process as metabolites [1, 2]. In the case of herbivore or pathogen attack, the emission amounts of certain volatile compounds, such as terpenoids, phenylpropanoids, fatty acid derivatives, and amino acid derivatives increase significantly. Among these compounds, terpenes are accounted for a large part and play a specific role in serving as attractants or deterrents for herbivores [3, 4]. The releasing amounts and species of volatile terpenes by plants at different growth stages have been considered as indicators of biological states to analyze their growth status [5]. One of commonly applied terpene detection techniques is gas chromatography (GC) or gas chromatography mass spectrometry (GC-MS), which is realized through separating gas molecules according to their chemical properties and detecting ionized gas fragments. This instrumental method shows the relatively high sensitivity and precision. In addition, QCM sensors network combined with the molecular imprinting technique are also applied for terpene detection [6]. Practically, terpene detection in agriculture requires transducers having the characteristics of realtime, high accuracy, and ability to tolerate the interference from the real field. However, the present terpene detection techniques are limited in the lab state.

Noble metal NPs such as AuNPs exhibit a strong absorption band different from spectrum of bulk metal. This absorption occurs when the incident photon frequency is resonant with the collective oscillation of the conduction electrons, which is known as the localized surface plasma resonance (LSPR) [7-11]. Based on this phenomenon, recent efforts toward controlling the physical and chemical properties of nano-structured materials through molecular-level design have generated enormous interest. The optical and electronic properties of such films can be tuned by varying the size of the metal particles or the molecular weight and/or structure of the organic component [12-13]. The optical and electrical properties of layer-by-layer self-assembled films comprised of Au or Ag NPs and various organic compounds have been investigated as well [1417]. Meanwhile, other reports show that the selectivity of such sensors can be tuned by introducing chemical functionality into the organic shell encapsulating the nanoparticles [14-17]. The selectivity of such sensors was found to be controlled by the solubility properties and the size of the linkers used for film preparation.

The goal of our present research was to investigate the dependence of vapor-sensing properties of alkanedithiol-interlinked gold nanoparticle films on the molecular structure, conductivity, and depend on the alkyl chain length. The films were prepared via layer-bylayer self-assembly using AuNPs solution. As alkanedithiol compounds, 1,4-benzene dithiol and 1,10-decanedithiol were used. The local refractive index sensitivity of films was investigated by sensing the object terpenes $\beta$ caryophyllene, $\alpha$-pinene, and myrcene while monitoring their LSPR spectra wavelength shift 
and analyzing the selectivity between linkers and target terpene gases.

\section{Experimental Section}

\subsection{Preparation of AuNPs solution}

Solutions of AuNPs in toluene were prepared as described by Brust and coworkers [13]. $30 \mathrm{~mL}$ of $30 \mathrm{mM}$ aqueous solution of hydrogen tetrachloroaurate was mixed with $80 \mathrm{~mL}$ of a $50 \mathrm{mM}$ solution of tetraoctylammonium bromide in toluene and stirred vigorously for at least $1 \mathrm{~h}$, and then waited for stratification. Extracted the organic phase tetrachloroaurate and slowly poured $25 \mathrm{~mL} \quad 0.5 \mathrm{M}$ sodium borohydride aqueous solution with vigorous stirring, after that, the ruby-colored organic phase was separated, washed once with $0.1 \mathrm{M}$ sulfuric acid, five times with water, respectively. The final solution was thoroughly dried with anhydrous magnesium sulfate. Au nanoparticles solution was diluted to $\sim 1000 \mathrm{mg} / \mathrm{L}$ before use.

2.2 preparations of self-assembled multilayer Au nanoparticle films on glass substrate

Prior to nanoparticles deposition, the glass substrates were cut to $26 * 10 \mathrm{~mm}$ and cleaned thoroughly with detergent, distilled water, acetone, and 2-propanol in a ultrasonic cleaner and dried by $\mathrm{N}_{2}$ stream, respectively. After that, the glass substrates were processed in a plasma cleaner (PDC-001, HARRICK PLASMA) in Argon atmosphere for $5 \mathrm{~min}$. Then the substrates were placed in a 1:12.5 (volume ratio) solution of 3-aminopropyl triethoxysilane (APTES) (Shin-Etsu Chemical) in toluene for 1 $\mathrm{h}$, rinsed thoroughly with toluene to remove physically adsorbed APTES molecules, and directly immersed into freshly prepared $\mathrm{Au}$ nanoaprticles solution overnight. Thoroughly rinsed with toluene and immersed in a $2 \mathrm{mM}$ dithiol toluene solution for $1 \mathrm{~h}$. Dithiol used in this study were 1,4-benzenedithiol and 1,10decanedithiol without further process. Subsequently, layers were deposited alternately immersion in AuNPs and dithiol solution for $3 \mathrm{~h}$ and $1 \mathrm{~h}$, respectively.

\subsection{UV-vis Spectrum}

The transmittance spectrum was obtained by an UV-vis spectrophotometer (UV1800, Shimadzu), in which the scanning range was set from 400 to 1000 nanometer, and the wavelength resolution was 0.5 nanometer, using air as baseline. The schematic experimental setup is shown in Fig. 1.

\subsection{Atomic force microscopy images}

The atomic force microscopy (AFM) images were collected on a Digital Instruments Nanoscope scanning probe microscope (SPM) operating in tapping mode for the morphology analysis of AuNPs. Pointprobe-silicon SPMsensor (Digital Instruments, Nanosensors) were used. Typical technical data include: thickness: $4 \mu \mathrm{m} /$ length: $125 \mu \mathrm{n} /$ width: $30 \mu \mathrm{m}$; resonance frequencies $320 \mathrm{kHz}$ / force constant: $42 \mathrm{~N} / \mathrm{m} /$ coating: none.

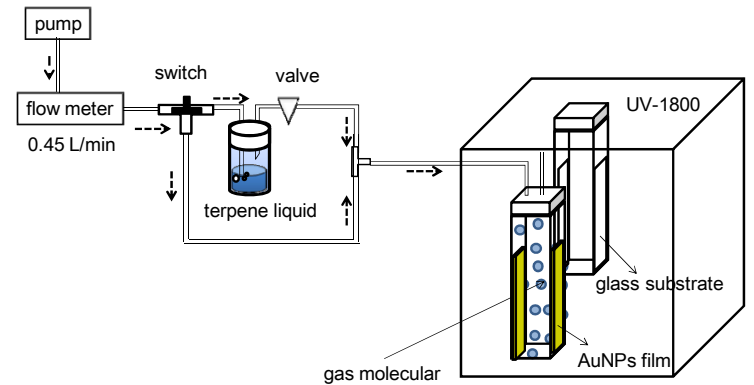

Fig. 1. Schematic experimental setup

\section{Results and discussion}

\subsection{UV-vis spectra of AuNPs films}

The UV-vis spectra of layer by layer structured AuNPs films are shown in Fig. 2.

(a)

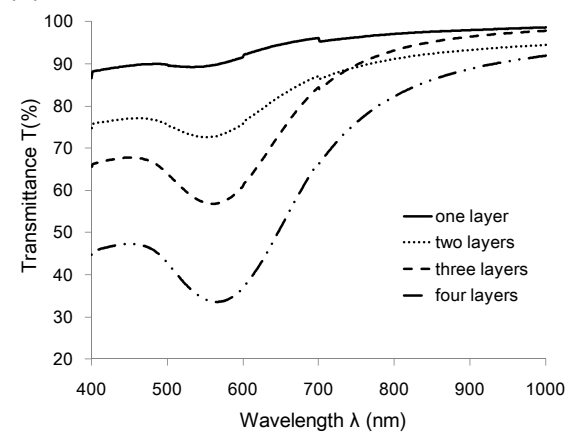

(b)

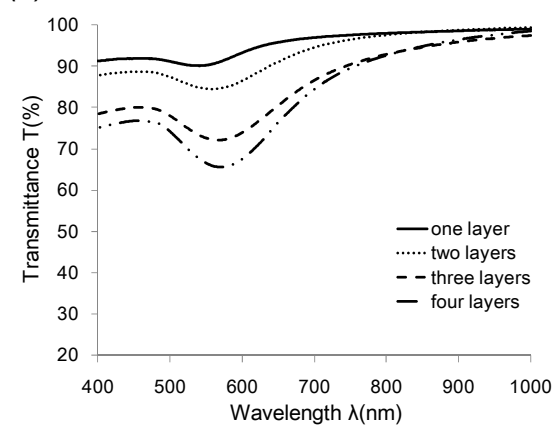

Fig. 2. UV/vis spectra layer by layer structured AuNPs linked with (a) 1,4-benzenedithiol and (b) 1,10-decanedithiol

The spectra of four consecutive layers linked with 1,4-benzenedithiol and 1,10-decanedithiol both show no further broadening and loss of peak features after the deposition of 4 layers, and wavelength of the lowest point of transmittance spectrum of 1,4-benzenedithiol and 1,10-decanedithiol shift from $\sim 530 \mathrm{~nm}$ to $\sim 570 \mathrm{~nm}$ and $\sim 565 \mathrm{~nm}$ with layer number 
increase, respectively, which can be attributed to the molecular electrical conductive character of these two dithiols, conductance of 1,4benzenedithiol is much larger than 1,10decanedithiol, so the spectral lowest point wavelength shift is larger for film with linker 1,4benzenedithiol than 1,10-decanedithiol.

The other difference is the tendency of transmittance variation of Au nanoparticles films linked with 1,4-benzenedithiol and 1,10decanedithiol, which can be attributed to particles accumulation phenomenon. The schematic two-layer AuNPs films linked by 1,4benzenedithiol and 1,10-decanedithiol are shown in Fig. 3.

(a)

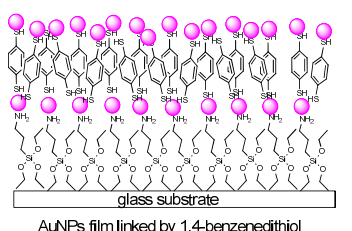

(b)

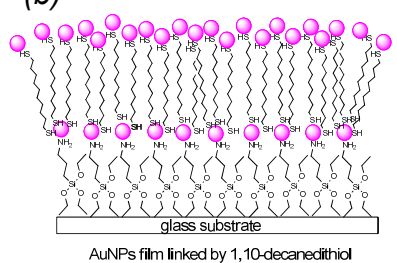

Fig. 3. The schematic two layer AuNPs film

The density of AuNPs increase with layer number increase, and AuNPs density linked by 1,10-decanedithiol is higher than AuNPs film linked by 1,4 -benzenedithiol. Comparing the color of four layer structured 1,4-benzenedithiol and 1,10-decanedithiol linked $\mathrm{Au}$ films, 1,4benzenedithiol linked film is light purple while the color of 1,10-decanedithiol linked Au films is dark purple. Spectral similarity is that both with longer cross-linking molecules 1,10decanedithiol and shorter ring-structured 1,4benzenedithiol linked AuNPs multilayers show linear transmittance decrease in the visible region from $400 \mathrm{~nm}$ to $1000 \mathrm{~nm}$ as a function of colloid coverage. However, according to the spectral difference, we concluded that the molecular length and their electrical resistance commonly decided their spectral characters.

3.2 The local refractive index sensitivity of layer by layer structured AuNPs

Layer by layer structured AuNPs films were used to detect three kinds of typical terpene gases, including $\beta$-caryophyllene, $\alpha$-pinene, and myrcene gas. The dynamic gas making system using bubbling method was used to generate target gases with a constant concentration. According to the liquid quantity difference before and after the bubbling process, the rough gas concentration of $\alpha$-pinene, $\beta$ caryophllene, myrcene were calculated to be about 4900 ppm, 90 ppm, 1500 ppm, respectively.

The average LSPR local refractive index sensitivity (the wavelength shift of lowest point in transmittance spectrum, $\Delta \lambda$ ) of AuNPs films is shown in Fig. 4.

(a)

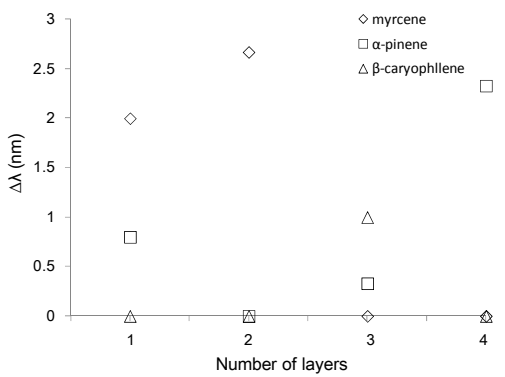

(b)

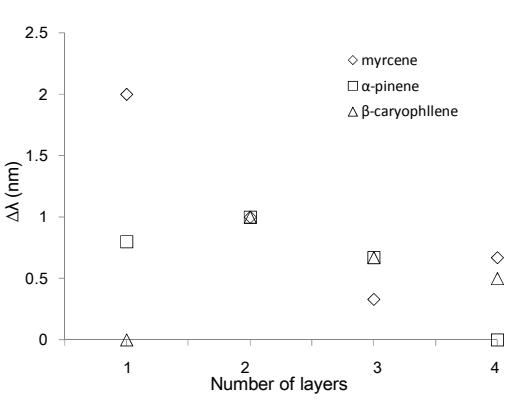

Fig. 4. LSPR sensitivity of layer by layer structured AuNPs linked with (a) 1,4-benzenedithiol and (b) 1,10-decanedithiol

The initial sensitivity values detected using selfassembled AuNPs (monolayer AuNPs) are different to three kinds of terpenes because of different terpene gas possessing different refractive index and volatility, etc. although all the terpene gases are generated with the same bubbling flow rate. An AFM image of monolayer AuNPs is shown in Fig. 5. The AuNPs' density is high, so AuNPs accumulation takes effects on the final sensitivity, and will be more conspicuous with layer number increase.

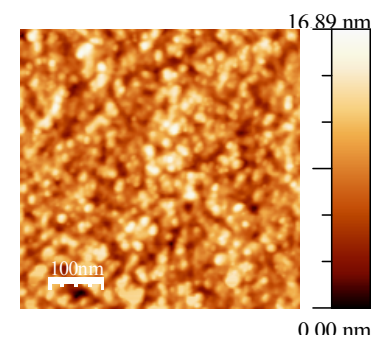

Fig. 5. AFM image of AuNPs monolayer

In this study, target terpene molecule is of nonpolarity. Correspondingly, 1,4-benzenedithiol and 1,10-decanedithiol also form non-polar linkers between AuNPs. Hence, the mutual solubility between terpenes and linkers seems to be good. As a result, these layer by layer structured AuNPs films were dosed with $\beta$ caryophyllene, $\alpha$-pinene, and myrcene molecular, when placed in terpene gases environment, respectively. Specifically, seen from figure 4 (a), 1,4-benzenedithiol linked $\mathrm{Au}$ 
nanoparticle films show certain selectivity to $\alpha$ pinene when the layer number increased to 4 . While 1,10-decanedithiol linked Au nanoparticle films show selective dissolution tendency to $\alpha$ pinene and $\beta$-caryophllene, which is conspicuously shown in films with 3 and 4 layers in figure 4 (b). In summary, the affinity between the film linker and terpene gas molecular commonly regulated their mutual solubility, and decides the final sensitivity of AuNPs films.

\section{Conclusion}

Methodology to generate films composed of AuNPs assembled in a stepwise fashion entirely from solution is presented. Thus, terpene gas detection based on the LSPR spectrum of this layer by layer structured AuNPs films linked respectively by 1,4benzenedithiol and 1,10-decanedithiol was realized. The experiment were carried out based on the fact that 1,4-benzenedithiol and 1,10-decanedithiol are different in molecular length and electrical resistance. Through comparing film's spectral character and sensitivity $(\Delta \lambda)$, we gained that linkers take effects on structural charecter of $\mathrm{Au}$ nanoparticle films. In addition, through analyzing sensitivity of AuNPs films to target terpene gases, we concluded that the selective dissolving ability of dithiols decide the final sensitivity. Studies to further enhance the sensitivity of this AuNPs films are underway.

\section{Acknowledgement}

This work is partly supported by the Strategic Information and Communications R\&D Promotion Program, Ministry of Internal Affairs and Communications.

\section{References}

[1] G. Arimura, C. Kost, Herbivore-induced, indirect plant defences, Biochimica et Biophysica Acta. 1734, 91-111 (2005); doi: 10.1016/j.bbalip.2005.03.001

[2] Turlings TCJ, Bernasconi M, The induction of volatile emissions in maize by three herbivore species with different feeding habits: possible consequences for their natural enemies, Biological Control: Theory and Applications in Pest Management 11, 122-129 (1998). doi: 10.1006/bcon.1997.0591

[3] T. Kollner, C. Schnee, The sesquiterpene hydrocarbons of maize (Zea mays) form five groups with distinct developmental and organspecific distributions, Phytochemistry 65, 18951902 (2004); doi: 10.1016/j.phytochem. 2004. 05. 021

[4] Z. Yan, C. Wang, Wound-induced green leaf volatiles cause the release of acetylated derivatives and a terpenoid in maize,
Phytochemistry 67, 34-42 (2006); doi:10.1016/j.phytochem. 2005.10. 005

[5] W. H. O. Ernst and P. J. Peterson, The role of biomarkers in environmental assessment (4). Terrestrial plants, Ecotoxicology 3, 180-192 (1994); doi: 10.1007/BF00117083

[6] Peter A. Lieberzeit, A. Rehman, QCM sensor array for monitoring terpene emissions from odoriferous plants, Monatsh Chem 140, 947-952 (2009); doi: 10.1007/s00706-009-0141-7

[7] M. Rycenga, Claire M. Cobley, Controlling the Synthesis and Assembly of Silver Nanostructures for Plasmonic Applications, Chem. Rev. 111, 3669-3712 (2011); doi: 10.1021/cr100275d

[8] Kathryn M. Mayer, Jason H. Hafner, Localized Surface Plasmon Resonance Sensors, Chem. Rev.111, 3828-3857 (2011); doi: 10.1146/annurev.physchem.58.032806.104607

[9] Gregory V. Hartland, Optical Studies of Dynamics in Noble Metal Nanostructures, Chem. Rev. 111, 3858-3887 (2011); doi: 10.1021/cr1002547

[10] Katherine A. Willets, Richard P. Van Duyne, Localized Surface Plasmon Resonance Spectroscopy and Sensing, Physical Chemistry 58, 267-297 (2007); doi: 10.1146/annurev.physchem.58.032806.104607

[11] B. Chen, C. Liu, Technical Digest of $28^{\text {th }}$ Sensor symposium, p5-6,pp. 663-666, 2011.

[12] Michael D. Musick, Christine D. Keating, Metal Films Prepared by Stepwise Assembly. 2. Construction and Characterization of Colloidal $\mathrm{Au}$ and Ag Multilayers , Chem. Mater. 12, 28692881 (2000); doi: 10.1021/cm990714c

[13] M. Brust, D. Bethell, Christopher J. Kiely, and David J. Schiffrin, Self-Assembled Gold Nanoparticle Thin Films with Nonmetallic Optical and Electronic Properties, Langmuir 14, 54255429(1998); doi: 10.1021/la980557g

[14] Y. Joseph, I. Besnard, Self-Assembled Gold Nanoparticle/Alkanedithiol Films: Preparation, Electron Microscopy, XPS-Analysis, Charge Transport, and Vapor-Sensing Properties. J. Phys. Chem. B 107, 7406-7413 (2003); doi: 10.1021/jp030439o

[15] J. Ye, K. Bonroy, Enhanced localized surface Plasmon resonance sensing on threedimensional gold nanoparticles assemblies, Colloids and Surfaces A: Physicochem. Eng. Aspects 321, 313-317 (2008); doi: 10.1016/j.colsurfa.2008.01.028

[16] W. Peter Wuelfing, Stephen J. Green, Electronic Conductivity of Solid-State, Mixed-Valent, Monolayer-Protected Au Clusters, J. Am. Chem. Soc. 122, 11465-11472 (2000); doi: 10.1021/ja002367

[17] D. Bethel, M. Brust From monolayers to nanostructured materials: an organicchemist's view of self-assembly, Journal of Electroanalytical Chemistry 409, 137-143 (1996); doi: 10.1016/0022-0728(96)04533-0 